DOI: https://doi.org/10.46296/gt.v3i6.0017

\title{
HALLAZGOS IMAGENOLÓGICOS DEL CARCINOMA ESCAMOCELULAR DE LENGUA: ESTUDIO DE CASO
}

\section{IMAGENOLOGICAL FINDINGS OF SQUAMOUS CELL CARCINOMA OF TONGUE: CASE STUDY}

\author{
Andrade-Bravo Galo Javier ${ }^{1 *}$ \\ ${ }^{1}$ Universidad Laica Eloy Alfaro de Manabí, ULEAM. Manta, Ecuador.
}

*Correo: galo.andrade18@gmail.com

\begin{abstract}
Resumen
El carcinoma epidermoide se encuentra entre las neoplasias más frecuentes, de manera que ocupa el lugar 12. Etiológicamente, se encuentra asociado con factores como el tabaquismo, consumo de alcohol, virus de inmunodeficiencia humana y sífilis. Además, el aporte de herramientas como la tomografía o resonancia magnética en el diagnóstico de enfermedades permite la detección de tumores malignos en etapas oportunas. En este trabajo, se presenta el diagnóstico y le evolución de un carcinoma epidermoide en un paciente de 47 años, con hábito de consumo de alcohol y tabaco. La resonancia magnética y la tomografía computarizada permitieron al médico emitir un diagnóstico de cáncer de lengua, cuya lesión ocupante, de dimensiones $86 \times 71 \times 48 \mathrm{~mm}$, se extendió alrededor de un $20 \%$.
\end{abstract}

Palabras clave: carcinoma epidermoide, diagnóstico, tomografía, resonancia magnética.

\begin{abstract}
Squamous cell carcinoma is among the most frequent malignancies, so it is ranked 12 . Etiologically, it is associated with factors such as smoking, alcohol consumption, human immunodeficiency virus and syphilis. In addition, the contribution of tools such as tomography or magnetic resonance imaging in the diagnosis of diseases allows the detection of malignant tumors at opportune stages. In this work, the diagnosis and evolution of an epidermoid carcinoma in a 47-year-old patient with an alcohol and tobacco consumption habit are presented. The magnetic resonance together with the computed tomography allowed the doctor to issue a diagnosis of cancer of the tongue, whose occupant lesion, with dimensions of $86 \times 71 \times 48 \mathrm{~mm}$, extended around $20 \%$.
\end{abstract}

Keywords: squamous cell carcinoma, diagnosis, tomography, magnetic resonance.

Información del manuscrito:

Fecha de recepción: 25 de marzo de 2020

Fecha de aceptación: 09 de julio 2020

Fecha de publicación: 10 de julio de 2020 


\section{Introducción}

Entre los casos más comunes de neoplasia maligna que pueden llegar a desarrollarse en la cavidad bucal, el carcinoma epidermoide es el más frecuente, puesto que se origina en los epitelios que recubren la mucosa y afecta, principalmente, el labio inferior, los bordes laterales de la lengua y el suelo de la boca (MezaGarcía et al., 2009). Además, el cuadro clínico de este proceso carcinogénico se caracteriza por la presencia de placas blancas, placas entre eritematosas y blancas, úlceras de bordes levantados y masas exofíticas (Liang et al., 2008).

Es así, que el carcinoma epidermoide de cavidad bucal ocupa el número 12 de todas las neoplasias malignas en el mundo, con variación de prevalencia de acuerdo con la región. En Estados Unidos se calcula que se presentan 12.000 nuevos casos al año, y representa el $23,3 \%$ de todas las neoplasias malignas de cavidad bucal. En México, la Secretaría de Salud menciona que de las defunciones (5327) causadas por neoplasias malignas en 1999, el $0,7 \%$ son de cavidad bucal (MezaGarcía et al., 2009).
Cerca de 18.000 personas al año desarrollan cánceres de la lengua y la boca en el mundo, de los cuales el $95 \%$ de los casos se refiere a carcinomas de células escamosas, y el resto corresponde a carcinoma verrugoso y tumores de las glándulas salivales menores. Generalmente, el $50 \%$ de los tumores de la boca se localizan en la lengua y la edad promedio de estos casos es de 60 años, con una proporción hombre mujer de 3:1, respectivamente (Ramírez et al., 2001).

En los últimos años se registra un incremento en el número de mujeres afectadas, probablemente relacionado al creciente hábito de fumar. Esta enfermedad es más frecuente en la raza blanca que en la negra (Regezi \& Sciubba, 2000). Referente a los niños, el cáncer de lengua en menores de 15 años es sumamente infrecuente. Tal es el caso de Finlandia, en donde se han 3 casos de carcinoma epidermoide entre 1961 y 1980 (Guidobono et al., 2011).

Por su parte, en Ecuador, según Fornell \& Villao (2005), existe un alto índice de mortalidad por cáncer, ocupando el segundo lugar, con 
relación a otras enfermedades catastróficas. Los tumores malignos de cavidad oral representan el $6 \%$ de todos los cánceres diagnosticados y el $30 \%$ de todos los tumores malignos de cabeza y cuello. El carcinoma de lengua ocupa el segundo lugar entre los tumores de cavidad oral, representando el $22 \%$ (Guidobono et al., 2011). En este marco, Meza-García et al. (2009), han mencionado diversos factores de riesgo asociados, como: el tabaquismo, alcoholismo, sífilis, infecciones por virus del papiloma humano, liquen plano bucal, VIH, irritantes mecánicos, higiene bucal deficiente, factores hereditarios, entre otros.

Desde su perspectiva, la tomografía computarizada (TC) es una técnica radiológica que consiste en el uso de rayos $X$ para producir imágenes transversales detalladas de un cuerpo. Sin embargo, a diferencia de los rayos $X$ regulares, la TC crea imágenes con detalles de los tejidos blandos y órganos. Este estudio permite a los médicos estimar el tamaño y la localización de un tumor, si se ha extendido a los tejidos cercanos, o si se ha propagado a los ganglios linfáticos del cuello. Se lo realiza, también, para determinar si hay propagación de cáncer en los pulmones (Alteri et al., 2018).

En TC, los signos radiológicos comunes y que se utilizarán como detección primaria de la lesión fueron la ocupación de espacio, ganglios afectados o calcificados, unidades Hounsfield al estudio simple y a la captación de contraste, presencia de metástasis a nivel óseo en columna cervical (García-Kass et al., 2013).

Al igual que la TC, las imágenes por resonancia magnética (RM) muestran detalles de los tejidos blandos del cuerpo, a pesar de que utilizan ondas de radio e imanes potentes en lugar de rayos $X$. Debido a que la RM provee una imagen detallada, es probable que se haga uso de esta técnica radiológica para determinar si hay propagación de cáncer en el cuello. Estos estudios también pueden ser muy útiles para observar otras áreas del cuerpo, especialmente el encéfalo y la médula espinal (Alteri et al., 2018).

Uno de los factores pronósticos independientes para la supervivencia y superación del cáncer de lengua es el espesor tumoral, el cual predice el riesgo de 
aparición de metástasis cervical.

Diferentes autores han evaluado la correlación de la medida del espesor radiológico en el cáncer de lengua en el estudio prequirúrgico, respecto al espesor anatomopatológico tras la resección tumoral mediante tomografía RM (Sánchez, 2015). De tal manera, se ha establecido que un espesor tumoral mayor a once milímetros predice la metástasis cervical hasta en un $93 \%$ de los casos en los pacientes diagnosticados con cáncer de lengua. Por su parte, la medida del espesor en estadios precoces es crucial para determinar la actitud terapéutica en estos pacientes (Brener et al., 2007).

Normalmente, el procedimiento realizado para confirmar o descartar el diagnóstico de cáncer de lengua consiste en un examen a conciencia de la zona de la boca y la faringe. Las dos terceras partes anteriores de la lengua pueden evaluarse a simple vista y la base de la lengua puede ser observada con la ayuda de un espejo. Cuando se sospecha que existe cáncer de lengua, el médico toma una muestra de tejido (biopsia) de las zonas de la piel alterada, para así asegurar el diagnóstico
(Manzano, 2001). A continuación, el médico empleará procedimientos de exploración por imágenes como la TC o la RM. De este modo puede determinarse la presencia de tumores ramificados en los ganglios linfáticos de la garganta. Otra opción viable consiste en la exploración mediante ultrasonido (GallegosHernández, 2016).

En cuanto al contexto legal, el Estado ecuatoriano garantizará la atención prioritaria a pacientes con enfermedades catastróficas o de alta complejidad, tal como lo indica la Constitución del Ecuador, la cual en su sección séptima dispone de acuerdo al Artículo 32 que "La salud es un derecho que garantiza el Estado, cuya relación se vincula al ejercicio de otros derechos; La prestación de los servicios de salud se regirá por principios de equidad, universalidad, solidaridad, interculturalidad, calidad, eficiencia, eficacia, precaución y bioética, con enfoque de género y generacional". Además, en su Artículo 50 dispone que "El estado garantizará a toda persona que sufre de enfermedades catastróficas o de alta complejidad el derecho a la atención especializada y gratuita en todos los niveles, de 
manera oportuna y preferente" (Constitución de la República del Ecuador, 2008).

En base a lo mencionado anteriormente, desde el Estado se garantiza que los Centros de Salud cuenten con los equipos de imágenes adecuados, como el tomógrafo y rayos $\mathrm{X}$. Además, el Estado Nacional tiene convenios con diferentes instituciones de Salud que poseen unidades de imágenes radiológicas, donde se incluyen tecnología para el estudio de RM. De esta manera, un diagnóstico adecuado es viable debido a la posibilidad de que los pacientes se sometan a los análisis correspondientes, como antecedente a un diagnóstico final con estudios complementarios.

Por ende, la importancia del presente estudio radica la necesidad de explicar la utilidad de los estudios imagenológicos en el diagnóstico del carcinoma escamocelular de lengua.

Para lo cual, se requiere una descripción de las técnicas imagenológicas de mayor impacto en el diagnóstico oportuno del carcinoma escamocelular de lengua. De esta manera, se podrá establecer el método imagenológico más eficaz, así como identificar signos radiológicos característicos de esta enfermedad.

\section{Metodología}

\subsection{Descripción del caso de estudio}

En este trabajo se expone el caso clínico de un paciente de sexo femenino de 47 años, de origen norteamericano y que reside en la parroquia Crucita del cantón Portoviejo. Entre sus antecedentes patológicos personales se evidencia que se le ha sido diagnosticado diabetes, pero no existe registro de enfermedades tumorales ni adenopatías en ninguna otra región del cuerpo. Sin embargo, en sus antecedentes familiares se indica que su padre fue diagnosticado con cáncer de pulmón a los 60 años de edad. Además, entre sus hábitos se menciona el consumo de tabaco, ingesta de bebidas alcohólicas de forma esporádica y la falta de ejercitación física de manera regular.

Dentro del procedimiento diagnóstico se registra el ingreso de la paciente al Subcentro rural de Salud de la parroquia Crucita debido a la presencia de una pequeña masa en borde izquierdo del suelo de la 
boca, así como la presencia de estado febril y frecuencia cardíaca elevada. A continuación, el personal médico del Subcentro evalúa y deriva a la paciente al área de odontología. Posterior a la revisión del odontólogo se determina la ausencia de lesión significativa en las piezas dentales. Debido al malestar presentado en la región examinada, el médico tratante receta la administración de analgésicos.

Debido a la evidencia de aumento de la masa en la zona afectada, la paciente ingresa al área de emergencia del hospital oncológico "Dr. Julio Villacreses Colmont" (SOLCA-Manabí) por sospecha de tumoración, en donde es atendida por un médico general, el cual realiza la exploración física por región. En la región craneal se observa normocéfalo, presencia de pupilas normales y ausencia de alteraciones a nivel del cráneo. En la región del cuello se evidencia cierta inflamación del cuello debido a la presencia de "masa palpable" en su cara superior izquierda; mientras que en la cara total derecha no se evidencia anormalidad. Además, se manifiesta la presencia de dolor al realizar movimientos de flexión, extensión y movimientos de deglución. En la región torácica se observa área simétrica, presencia de prótesis mamarias en ambas mamas, campos pulmonares claros y ventilados, ruido cardíaco presente ligeramente aumentado, no se presentan signos de lesión de tórax o presencia de masa anormal de pared. En la región abdominal no se evidencian hematomas o cambio de color en la piel, a la palpación se halla la presencia blando depresible, así como de ruidos hidroaéreos. La la región pélvica se encuentra sin cambio de coloración en la piel, además esta se halla fija e inmóvil. La revisión tanto en extremidades superiores como inferiores indica que ocurre movimiento normal de las extremidades y que no existe evidencia de alguna lesión. Los signos vitales de la paciente se encuentran dentro de los rangos normales, sin embargo, presenta dificultad para hablar debido a la lesión que presenta en su boca. De esta manera, el médico encargado requiere la valoración urgente mediante exámenes clínicos y paraclínicos, donde se determinan los siguientes valores: Glucosa: 125 $\mathrm{mg} / \mathrm{dL}$, Urea $24 \mathrm{mg} / \mathrm{dL}$, Creatinina $0,71 \mathrm{mg} / \mathrm{dL}$, Biometría Hemática, 
Neutrófilo Segmentado: $94 \%$, Linfocitos 4,4\%, Monocitos: 1,6\%,

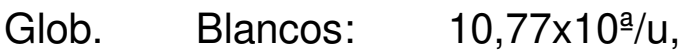
Eritrocitos: 4,26 10"6/4, Plaquetas: 204x 10\%/u, Hemoglobina: $12.8 \mathrm{~g} / \mathrm{dL}$, Hematocrito: $38 \%$, Tiempo Parcial Tromboplastina: $26,7 \mathrm{seg}$, Testigo TTP: 22,2 seg, Tiempo de Protombina: 10,9seg, \% de activación: 112,9\%, INR: 0,91, Electrolitos, Sodio: $140 \mathrm{mmol} / \mathrm{L}$, Potasio: 4,40mmol/L, Cloro: 107,9 $\mathrm{mmol} / \mathrm{L}$.

Debido al elevado nivel de glucosa y a la sospecha de lesión tumoral en la cavidad oral, la paciente es ingresada para la obtención de hallazgos radiológicos, por lo que se ordenan estudios de TC de cuello con contraste para evidenciar localización de la masa, valoración de estructuras cervicales, suelo de la boca, glándulas adyacentes, densidades Hounsfield antes $y$ durante la captación de contraste. Además, se recomienda un estudio de RM simple y con contraste para valorar la masa en parte blanda, la lesión ocupante del espacio o región, tejidos afectados, glándulas afectadas, captación de contraste en la lesión, y progresión de la masa con relación al estudio anterior. Los estudios fueron realizados en fecha del 27 de febrero del 2018 y 15 de marzo del 2018, respectivamente, mediante equipos de imagenología en la unidad hospitalaria SOLCA Manabí.

El estudio posterior de RM de cuello indica que en la glándula submaxilar izquierda se evidencia lesión ocupante de espacio cuyas medidas fueron $41 \times 38 \mathrm{~mm}$, con aumento de volumen de aproximadamente el $20 \%$ en comparación al estudio anterior, de bordes mal definidos.

Una vez que se realiza el análisis de los resultados del estudio de imagen, se ordena realizar el procedimiento de eco guías para biopsias de partes blandas, con el propósito de estudiar microscópicamente las muestras de masa, de manera que el departamento de patología emita un informe final de la enfermedad.

Producto del aumento de la tumoración se coloca una sonda de alimentación parenteral a la paciente, debido a la complicación en la ingesta de alimentos. El 17 de abril del 2018 se realiza una placa de rayos $\mathrm{X}$ simple de abdomen para valorar órganos abdominales y si existe presencia de adenopatías. 
Posteriormente, la paciente es derivada al área de radioterapia, para cuidados paliativos. Se determina que la tumoración de cavidad oral se encuentra en estadio T4 "tumor mayor a $4 \mathrm{~cm}$ con invasión de estructuras adyacentes" y el departamento de cuidados paliativos solicita RM de cuello simple y con contraste, y TC de tórax simple. La TC simple de tórax es enviada para valorar campos pulmonares, bronquios principales y cada segmento pulmonar en caso de existir presencia de metástasis. La RM de cuello simple y con contraste es solicitada por el departamento de radioterapia para observar la progresión de la tumoración en la zona de la lengua y parte del cuello, donde se evidencia la invasión de ciertas zonas de músculos.

Debido a las condiciones progresivas de la lesión, en el mes de septiembre, la paciente es trasladada a casa para guardar reposo y continuar la etapa final de la enfermedad en compañía de sus familiares.

\section{2. Ámbito de estudio}

En relación a la información recopilada a través de los exámenes realizados para obtener un diagnóstico preciso, los ámbitos a intervenir en el estudio del presente caso corresponden a la valoración mediante exploración física e imagenológica de las regiones de la cabeza y cuello, conformada por la lengua (en su borde retro e infra mandibular izquierdo), la región torácica y abdominal con su capacidad total para verificar presencia de metástasis.

\subsection{Actores implicados}

En el estudio desarrollado en este trabajo se encuentran implicados tanto paciente y familiares, debido a que estos proporcionaron información relevante. Además, los médicos que trataron a la paciente, realizando exámenes para corroborar el diagnóstico presuntivo. Por su parte, los licenciados en imagenología que proporcionaron los estudios de imagen, los cuales colaboraron en la determinación del diagnóstico.

\subsection{Identificación del problema}

A pesar del aviso en campañas preventivas que exponen los efectos del tabaquismo y el consumo de alcohol, puesto que estos dos factores de riesgo combinados 
representan la principal causa de la formación del carcinoma escamoso de lengua, la sociedad hace caso omiso. Además, uno de los principales inconvenientes de no realizar el diagnóstico oportuno de una enfermedad es el desarrollo de un cuadro sintomatológico de modo silencioso, de manera que es importante que, para la identificación del carcinoma escamoso de lengua, la auto revisión del paciente sea efectuada ya sea por presentar una úlcera o erupción. Posteriormente, la evaluación clínica realizada por el médico, mediante una revisión física, identificará signos de enrojecimiento de la zona afectada con presencia de una masa nodular o irregular acompañada por secreciones en ciertos casos. Por último, para corroborar el diagnóstico, el principal método consiste en el estudio de RM, no obstante, en Manabí no se cuenta con equipamiento suficiente para la aplicación de un radiodiagnóstico eficaz, sumado a su alto costo.

\subsection{Fuentes de información}

Para el desarrollo de esta investigación se contó con la ayuda de un licenciado en radiología, quien colaboró con las diferentes imágenes de los estudios realizados al paciente; un médico radiólogo encargado de revisar las imágenes y emitir un informe; la historia clínica y datos recolectados por familiares del paciente; información recolectada de diferentes fuentes bibliográficas y tutorías del tutor de investigación en las diferentes etapas del desarrollo de este trabajo.

\subsection{Técnicas de recolección de información}

Las técnicas $y / 0$ instrumentos de investigación que se utilizaron en el desarrollo de esta investigación son la entrevista a profundidad dirigida a tres expertos en el área de imagenología, observación dirigida a las imágenes realizadas para el diagnóstico imagenológico y revisión documental dirigida al expediente clínico y revistas científicas, libros y otras fuentes bibliográficas.

\section{Resultados y discusión}

Los cánceres de la porción móvil de la lengua están situados por delante de la $\mathrm{V}$ lingual y corresponden a los dos tercios anteriores del órgano. Comprenden los bordes laterales, la cara dorsal y la cara ventral. Las neoplasias de la base de la lengua ocupan el tercio posterior de la 
lengua, por detrás de la $\mathrm{V}$ lingual. En algunos casos, la invasión tumoral masiva de la porción móvil de la lengua o de la totalidad de la lengua no permite determinar con certeza el lugar de origen del tumor (GarcíaKass et al., 2013).

La contribución de los estudios imagenológicos al diagnóstico clínico ha sido significativa. De esta manera, Boody \& Brook (2008) afirman que la RM ayuda a la identificación de tumores mediante la magnificación de las diferencias en el contenido del agua y flujo de sangre entre tejidos. Los tumores malignos crecen en su propia red de los vasos sanguíneos vía angiogénesis; esto le da una reserva de sangre más grande que la del tejido cercano. El material de contraste resalta la concentración de sangre en los vasos sanguíneos para ayudar a marcar los crecimientos malignos, cuando el agente contrastante es utilizado se puede valorar el tamaño y localización de crecimientos benignos o malignos, agrandamiento de los ganglios linfáticos, cambios en el flujo de la sangre, volumen extracelular, las cuales son ventajas de la RM para contribuir al diagnóstico del carcinoma escamocelular de lengua. Otro estudio indispensable y de primera mano que ayuda en el diagnóstico del carcinoma escamocelular de lengua es la TC. Según García-Kass et al. (2013), el escáner mediante TC es ampliamente utilizado como una ayuda para determinar en el preoperatorio el estadio de los ganglios del cuello y para evaluar la lesión primaria. Este estudio se ha convertido en el método estándar frente a otras técnicas de imagen producto de sus ventajas en costo y disponibilidad en los centros de salud. Esto se confirma según la entrevista realizada a expertos en el área de imagen, corroborando que la TC, junto a la RM son los estudios que contribuyen de manera directa para el diagnóstico de carcinoma escamocelular de lengua.

El estudio de TC de cuello con contraste, realizado el 27 de febrero del 2018, describe el proceso expansivo en el suelo de la boca izquierdo y glándula submaxilar izquierda, con densidad en los cortes simples en la pared central de 26 unidades Hounsfield (HU) y en la periferia de $46 \mathrm{HU}$. Con el medio de contraste se observó 
moderadamente en la periferia con densidad de $72 \mathrm{HU}$, e impresiona en la parte central con necrosis tumoral con densidad de los cortes contrastados de $36 \mathrm{HU}$. Además, el estudio no evidencia adenopatías cervicales y refleja el diámetro de vasos carotideos de calibre normal, tráquea normal, cuerpos vertebrales de forma y tamaño normal con su canal raquídeo y medula espinal de diámetro y densidad conservada.

Diferentes técnicas radiológicas han sido utilizadas para diagnosticar carcinoma escamo celular de lengua. De acuerdo con García-Kass et al. (2013), la RM nuclear se ha convertido en el método de referencia para identificar el carcinoma escamoso de lengua, el cual se suele presentar en pacientes con hábitos de tabaquismo y alcoholismo, la lesión puede ocurrir a diferentes niveles de esta estructura y podría invadir zonas aledañas a la misma, en el $75 \%$ suele ser una lesión maligna y en un $25 \%$ según el tratamiento podría presentar mejoría. Expertos en el área de imagen, manifiestan que la RM nuclear es el estudio fundamental para el diagnóstico de esta patología, confirmado con la observación de imágenes obtenidas en las distintas secuencias de la RM nuclear, realizadas al paciente objeto de estudio, comparándose la calidad de imágenes en tejidos blandos lo que no podría ser observado en otros estudios.

A partir de los resultados del estudio de RM nuclear simple más contraste, realizado el 15 de marzo del 2018, el médico imagenólogo informa el aumento de alrededor del $20 \%$ del volumen de lesión ocupante de espacio que en la fecha de estudio mide $41 \times 38 \mathrm{~mm}$ en la zona de la glándula submaxilar izquierda. La lesión, de bordes mal definidos, se extiende a nivel de planos del tendón digástrico, aumenta el efecto de masa, captación de contraste a predominio periférico y área central de necrosis, infiltración papilar del digástrico y obliteración del receso orofaringe ipsilateral.

Además, para obtener el diagnóstico histopatológico se le realizó al paciente biopsia guiada por ecografía. Según Martínez-Machuca et al. (2003), la biopsia guiada por ecografía es una técnica complementaria diagnóstica que utiliza ondas sonoras para ayudar a localizar un bulto o anormalidad y 
extraer una muestra de tejido para examinarla bajo microscopio. El médico utiliza la aguja para extraer la muestra de tejido para su análisis histológico, citológico, microbiológico o analítico, el procedimiento indica a estudios previos que se observa una lesión compleja en localización retro e infra mandibular izquierda, con área central de necrosis, se toma muestra líquida del área central, se realiza trucut de partes sólidas (2 cilindros).

Los resultados del departamento de patología confirman la sospecha del médico imagenólogo, resultado de biopsia tejidos blandos infiltrados por carcinoma escamocelular, se toman tres placas con muestra líquida de área central y esto solo confirma la sospecha de malignidad.

El objetivo de la biopsia es la obtención de material suficiente para conseguir diagnosticar la lesión u obtener información suficiente para definir el manejo del paciente o su pronóstico. Es menos invasiva que la biopsia quirúrgica, deja poco o nada de cicatriz, y no requiere de la exposición a la radiación ionizante. (Guevara, 2017)
Para el control y seguimiento de la sonda parenteral, se realizaron estudios de rayos $x$ simple de abdomen, observándose los siguientes hallazgos: sonda parenteral normoposicionada, silueta hepática y renal de tamaño y morfología normal, no se aprecia ascitis, marco óseo sin alteraciones. Según Boody \& Brook (2017) los rayos $\mathrm{X}$ abdominales utilizan una dosis muy pequeña de radiación ionizante para producir imágenes del interior de la cavidad abdominal. Se utilizan para evaluar el estómago, el hígado, los intestinos y el bazo, y se pueden utilizar para diagnosticar dolores inexplicables, náuseas o vómitos.

Diversos signos radiológicos característicos del carcinoma escamocelular de lengua se han evidenciado mediante RM. Los resultados mostrados por los estudios indican lesión ocupante de espacio, de densidad en secuencia T1, T2, FLAIR, difusión, Hipointensas o hiperintensas según la afectación y estadificación de la tumoración, relación de órganos, músculos y tejidos blandos con relación a la tumoración, valoración 
de estructuras con gadolinio (GarcíaKass et al., 2013)

En su última resonancia magnética de cuello simple más contraste se evidenciaron los hallazgos de nasofaringe normal, orofaringe a nivel posterior izquierdo de la base de la lengua y se observa lesión ocupante de espacio, que mide $86 \times 71 \times 48 \mathrm{~mm}$ de diámetro vertical, transverso y AP, de márgenes irregulares, heterogéneo a predominio hiperintenso y mixto a predominio sólido. Efecto de masa con desplazamiento de pared faríngea. La lesión capta contraste en zonas sólidas y restringe en la secuencia de difusión. Se observa marcado enlentecimiento a nivel de flujo vena yugular y seno sigmoideo ipsilateral. Tiroides normal. Glándulas submaxilares y parótidas: submaxilar izquierda no visualizada. El experto en imagen informa y sugiere que la lesión en comparación con exámenes anteriores se encuentra progresiva. Lo que se relaciona con la observación a las imágenes de RM nuclear realizadas al paciente, donde se detectaron signos radiológicos claves para el diagnóstico eficaz de esta patología.
En el último estudio de tomografía computarizada de tórax por control se evidencia el hallazgo de ganglios calcificados hiliares, el mayor de ellos mide $18 \mathrm{~mm}$ y en mediastino en ventana aortopulmonar de $10 \mathrm{~mm}$ de aspecto residual, granuloma calcificado en segmento lingular superior a $3 \mathrm{~mm}$ de aspecto residual, traqueostomía normoposicionada, estructuras mediastínicas de morfología normal, hilios pulmonares de aspecto normal, sin evidencia de tumoraciones, no se observan alteraciones de pared torácica, no se aprecia derrame pleural, como conclusión no hay signos de infiltración secundaria, traqueostomía normoposicionada, cambios residuales de enfermedad granulomatosa, lo cual es corroborado con el criterio del experto en imagen que manifiesta que la TAC como estudio control y de seguimiento permite visualizar signos radiológicos de los pulmones que la RMN no permite.

Aunque el diagnóstico de los tumores de cavidad oral está basado en la exploración física, los estudios de imagen de detección primaria o prequirúrgicos constituyen un pilar fundamental para establecer una 
correcta valoración de estos y optimizar su tratamiento (Lyon, 2013).

\section{Conclusiones}

El diagnóstico del carcinoma epidermoide se desarrolló a través de diferentes etapas. Inicialmente, se realizó un exploración física y exámenes clínicos para estimar las condiciones de la paciente. La resonancia magnética nuclear (RMn), es el estudio más frecuente para el análisis de procesos neoplásicos. Esta técnica permite determinar el espesor, y la densidad Hounsfield de las paredes del tumor. Las imágenes revelan que, la lesión ocupante de espacio presenta dimensiones de $86 \mathrm{~mm}$ de diámetro vertical, $71 \mathrm{~mm}$ de diámetro transversal y $48 \mathrm{~mm}$ de diámetro periférico, con densidades Hounsfield de 26 y 46 unidades en la pared central y en la periferia, respectivamente. A pesar de la aplicación del tratamiento de radioterapia, estudios posteriores de RMn revelaron que el crecimiento del tumor fue de alrededor del $20 \%$.

\section{Bibliografía}

Alteri, R., Kalidas, M., \& Yadao, L. (2018). American Cáncer Society. Chile. SERAM.
Asamblea Nacional Constituyente. (2008). Constitución de la República del Ecuador. Montecristi: Lexis.

Boody, R., \& Brook, O. (2017). Importancia de Rayos $X$ en Chest, Abdomen, Pelvis. Revista Radiológica, USA: Watson. p. 6.

Brener, S., Jeunon, F. A., Barbosa, A. A., \& Grandinetti, H. D. A. M. (2007). Carcinoma de células escamosas bucal: uma revisão de literatura entre o perfil do paciente, estadiamento clínico e tratamento proposto. Rev Bras Cancerol, 53(1), 63-9.

Fornell, G. U., \& Villao, V. (2005). Cáncer de lengua: Reporte de un caso. Medicina, 10(2), 130133.

Gallegos-Hernández, J. F. (2016). Metástasis ganglionares cervicales de carcinoma epidermoide con tumor primario desconocido. Abordaje diagnóstico terapéutico actual. Anales Médicos de la Asociación Médica del Centro Médico ABC, 61(1), 25-32.

García-Kass, A., DomínguezGordillo A., García-Núñez J., Cancela-Rivas G., TorresSalcines, J., \& EsparzaGómez, G. (2013). Revisión y puesta al día en cáncer de lengua. Avances en odontoestomatología, 29(5), 255-269. 
Guevara, D. E. (2017). Sociedad chilena de radiología. Chile: SOCHRADI, 23(3)

Guidobono, J., Evertz, M., Ríos, J., \& Zimmerman, J. (2011) Servicio de cirugía $y$ oncología Hospital José. R. Vidal - Corrientes. Argentina: SMRI.

Liang, X. H., Lewis, J., Foote, R., Smith, D., \& Kademani, D. (2008). Prevalence and significance of human papillomavirus in oral tongue cancer: the Mayo Clinic experience. Journal of Oral and Maxillofacial Surgery, 66(9), 1875-1880. https://doi.org/10.1016/j.joms. 2008.04.009

Lyon, A., (2013) Cáncer en España. Ministerio de Sanidad y Consumo. Cancer Incidence in Five Continents. Madrid, España: MARBAN, Vol. 5.

Manzano, A. C. D. M. (2001). Caracterização imunohistoquímica do infiltrado linfocitário em biópsia de carcinoma espinocelular da língua e soalho oral e sua implicação prognostica. Dissertação (mestrado) Universidade Estadual de Campinas, Faculdade de Ciências Médicas, Campinas, SP. Disponível em: $<$ http://www.repositorio.unica mp.br/handle/REPOSIP/3137 94>.
Martínez-Machuca, S., AlonsoBabarro, A., AparicioJabalquinto, G., Parga-Soler, N., \& Prieto-Checa, I. (2003). Cambios epidemiológicos en el cáncer epidermoide de lengua: A propósito de un caso. Medifam, 13(4), 84-88.

Meza-García, G., Muñoz-lbarra, J. J., Páez-Valencia, C., CruzLegorreta, B., \& AldapeBarrios, B. (2009). Carcinoma de células escamosas de cavidad bucal en un centro de tercer nivel de atención social en la ciudad de México: Experiencia de cinco años. Avances en odontoestomatología, 25(1), 19-28.

Ramírez, G., Patiño, F., Castro, J., Ramírez, C., Tejada, E., Muñoz, A., \& Mendoza, A., (2001). Guías de práctica clínica en enfermedades neoplásicas. Instituto Nacional de Cancerología. México: SERAM.

Regezi, J. A., \& Sciubba, J. (2000) Patología Bucal; Correlaciones clinicopatológicas. McGrawHill Interamericana.

Sánchez, C. (2015). Unidad de gestión clínica de cirugía oral y maxilofacial. Hospital Regional de Málaga. España. MARBAN 